\title{
MEDIR LA PROGRESIÓN DE LA COMPRESIÓN LECTORA EN LA ENSEÑANZA DE LENGUA EXTRANJERA PARA TRADUCTORES. UNA EXPERIENCIA DE EVALUACIÓN
}

\author{
Lupe Romero \\ Universitat Autònoma de Barcelona \\ Barcelona, Espanha
}

\begin{abstract}
Resumen: En este artículo presentaremos los primeros resultados de las pruebas de verificación de la adquisición de las lenguas extranjeras, en que se recogen datos de los niveles de comprensión lectora de la primera y la segunda lengua extranjera del grado de Traducción e Interpretación (GTI) de la Facultad de Traducción e Interpretación (FTI), que se imparte en la Universidad Autónoma de Barcelona (UAB). El objetivo principal de las pruebas es verificar si los niveles de lengua establecidos previamente en la implementación de las asignaturas del plan de estudios se corresponden con los niveles que poseen los alumnos al acabar de cursar dichas asignaturas. En ese sentido, describiremos el proceso de realización de las pruebas teniendo en cuenta los siguientes aspectos: características y desarrollo de las pruebas, características de la muestra, instrumento utilizado, resultados obtenidos y tendencias observadas. La finalidad última de las pruebas es asegurar una mejor formación del alumno ya que, a partir de los resultados obtenidos, podremos modificar o corregir los niveles de comprensión lectora de entrada y salida de las asignaturas y/o las actuaciones docentes y, por tanto, perfilar mejor la progresión en la adquisición de la competencia lectora de la lengua extranjera.
\end{abstract}

Palabras clave: Enseñanza de idioma en la formación de traductores e intérpretes; Adquisición de la comprensión lectora en lenguas extranjeras; Diseño curricular: progresión de la enseñanza 


\title{
MEASURING PROGRESS IN READING COMPREHENSION SKILLS IN FOREIGN-LANGUAGE TEACHING FOR TRANSLATORS. AN ASSESSMENT- BASED EXPERIENCE
}

\begin{abstract}
In this article we will present the first results of foreign-language acquisition verification tests performed by students of the Translation and Interpreting degree programme taught at the Faculty of Translation and Interpreting (FTI) of the Autonomous University of Barcelona (UAB), which provide data on the level of their reading comprehension skills in their first and second foreign languages. The tests are chiefly intended to check whether pre-established language levels for the subjects that are part of the syllabus match those actually attained by students upon completion of the subjects in question. We will describe the test process, including the characteristics of the tests and how they are carried out, the characteristics of the sample studied, the instrument used, the results obtained and the trends observed. The ultimate purpose of the tests is to improve the training students receive, as the results obtained will serve as a basis for changing or correcting the different subjects' initial and final reading comprehension skill levels and/or the approach taken by teaching staff, thus enabling us to better define progress in the acquisition of foreign-language reading comprehension skills.
\end{abstract}

Keywords: Language teaching in translator and interpreter education. Acquisition of reading comprehension skills in foreign languages. Curriculum design: teaching progression.

\section{Introducción}

En este artículo presentaremos los primeros resultados de las pruebas de verificación de la adquisición de las lenguas extranjeras, en que se recogen datos de los niveles de comprensión lectora de la primera y la segunda lengua extranjera del grado de Traducción e Interpretación (GTI) de la Facultad de Traducción e Interpretación (FTI), que se imparte en la Universidad Autónoma de Barcelona (UAB). El objetivo principal de las pruebas es verificar si los ni- 
veles de lengua establecidos previamente en la implementación de las asignaturas del plan de estudios se corresponden con los niveles que poseen los alumnos al acabar de cursar dichas asignaturas.

La finalidad última de las pruebas es asegurar una mejor formación del alumno ya que, a partir de los resultados obtenidos, podremos modificar o corregir los niveles de comprensión lectora de entrada y salida de las asignaturas y/o las actuaciones docentes y, por tanto, perfilar mejor la progresión en la adquisición de la competencia lectora de la lengua extranjera. De hecho, esta medida se enmarca en el proceso de "Seguimiento, Evaluación y mejora de las titulaciones", uno de los procesos del Sistema de Garantía Interno de Calidad (SGIQ) de la FTI, cuyo objetivo es garantizar la calidad y la mejora de la titulación en todos los procesos que intervienen en su gestión (acciones de orientación a los estudiantes, evaluación de los estudiantes, gestión de la movilidad, de las prácticas, etc.). Por ello, aunque los resultados que se presentan son los correspondientes a los cursos académicos 2011-2012 y 2013-1014, se prevé la realización de las pruebas de manera periódica.

Cabe destacar que las pruebas realizadas hasta ahora se han centrado en la comprensión lectora ya que de las cuatro habilidades posibles (comprensión lectora, comprensión oral, producción escrita y producción oral) es la comprensión lectora, la competencia fundamental en la formación en lengua para traductores (Berenguer 1996, 1997; Brehm 1997; Brehm Cripps y Hurtado Albir 1999). La traducción de textos escritos es la tarea profesional más frecuente para los traductores y, por ello, comprender el texto en lengua extranjera en profundidad es imprescindible para poder traducirlo.

\section{El grado de Traducción e Interpretación (GTI)}

El grado de Traducción e Interpretación de la UAB se comenzó a implementar en el curso académico 2009-2010, como sustituto de la anterior licenciatura de Traducción e Interpretación que se impartía en el mismo centro desde 1991. La transformación de la 
licenciatura en un Título de Grado responde a la necesidad de adaptar la formación académica de traductores e intérpretes a los requerimientos del Espacio Europeo de Educación Superior (EEES), cuyo objetivo principal es la convergencia en materia de educación de los países europeos ${ }^{1}$. Para conseguir dicho objetivo, las universidades europeas han tenido que transformar aspectos que tienen que ver con la adopción del crédito europeo ECTS como unidad de acreditación y transferencia, con la definición de competencias como parámetros de evaluación y con la implantación del sistema de evaluación continua para la mayoría de las asignaturas de las licenciaturas. En este sentido, la FTI contaba con la ventaja de que un sector importante del profesorado ya aplicaba metodologías activas, procedentes de la didáctica de lenguas y otras didácticas, y potenciaba el aprendizaje autónomo. De hecho, con anterioridad a la implantación del nuevo plan de estudios del actual grado de Traducción e Interpretación, la FTI impulsó un Plan Piloto de adaptación al EEES (2001-2008), cuya aplicación permitió afinar experiencias ya adquiridas y aplicar de modo eficaz las propuestas nacidas en el Espacio Europeo de Educación Superior.

\section{La formación en las lenguas extranjeras en el grado de Traducción e Interpretación (GTI)}

Los estudiantes del GTI estudian dos lenguas extranjeras a lo largo de los cuatro años que duran los estudios. La primera lengua extranjera (inglés, francés o alemán) suele ser un idioma que ya ha sido cursado a lo largo del bachillerato (o de estudios similares). Para acceder a los estudios, los estudiantes deben poseer en la primera lengua extranjera que deseen cursar (lengua B), un nivel mínimo correspondiente al nivel B1 (para francés y alemán) y B2 (para inglés) del Marco común europeo de referencia para las len-

${ }^{1}$ Para mayor información sobre el Espacio Europeo de Educación Superior, consulte < http://www.eees.es/ > .

Cad. Trad., Florianópolis, v. 38, $\mathrm{n}^{0}$ 2, p. 320-338, mai-ago, 2018 
guas: aprendizaje, enseñanza, evaluación (MCER)², cuyo conocimiento se comprueba mediante acreditación (en el caso de francés y alemán) o con las pruebas de aptitud previas a la matrícula (en el caso del inglés).

La segunda lengua extranjera (inglés, francés, alemán, portugués, italiano, ruso, árabe, chino, japonés) se trata de un idioma del que no se requieren conocimientos previos antes de iniciar los estudios universitarios y que se adquieren a lo largo de la carrera a partir del nivel inicial de principiante. Esta segunda lengua extranjera (lengua $\mathrm{C}$ ) debe complementarse a menudo con estancias en el país, sobre todo cuando la lengua elegida es filológicamente distinta de las lenguas maternas o lenguas A (que en el caso del GTI, son dos: el castellano y el catalán). Obviamente, la mayor o menor distancia filológica entre lenguas, implicará una diferente progresión en el aprendizaje de las lenguas extranjeras, ya que la adquisición del conocimiento de una lengua cercana (p.e: italiano o portugués) es mucho más rápida de lo que pueda ser entre lenguas lejanas (p.e: árabe o ruso). De hecho, mientras en las lenguas $\mathrm{B}$, los contenidos de lengua y traducción se concretan en asignaturas diferentes, en el caso de las lenguas $\mathrm{C}$, a excepción del primer curso donde los contenidos son únicamente de lengua, desde el segundo curso hasta cuarto, las asignaturas de lengua $\mathrm{C}$ presentan contenidos variables de lengua y traducción en función de la distancia filológica y la dificultad en el aprendizaje.

En el caso específico de las lenguas cercanas $\mathrm{C}$, objeto de estudio de este trabajo (inglés, francés, alemán, portugués e italia-

\footnotetext{
${ }^{2}$ El Marco Común Europeo de Referencia de las Lenguas forma parte del proyecto general de política lingüística del Consejo de Europa, cuyo objetivo principal es la unificación de directrices para el aprendizaje y la enseñanza de lenguas dentro del contexto europeo. En el MCER se describe de forma integradora lo que tienen que aprender a hacer los estudiantes de lenguas con el fin de utilizar una lengua para comunicarse. La descripción también comprende el contexto cultural donde se sitúa la lengua, los niveles de dominio de la lengua que permiten comprobar el progreso de los alumnos en cada fase del aprendizaje, y los niveles de dominio lingüístico exigidos por los exámenes y programas de evaluación existentes con el fin de facilitar las comparaciones entre distintos sistemas de certificados.
} 
no), las cuatro asignaturas de primer y segundo curso se dedican íntegramente a contenidos lingüísticos (Idioma C1 y C2; Idioma y Traducción C1 y C2); en tercero, las dos asignaturas se dedican exclusivamente a traducción (Idioma y Traducción C3 y C4) y en $4^{\circ}$ curso, una de las asignaturas se dedica a lengua (Idioma y Traducción C5) y otra a traducción (Idioma y Traducción C6).

En ese sentido, cabe destacar el trabajo realizado por la $\mathrm{Co}$ misión para la Implantación, seguimiento y evaluación del grado $G T I^{3}$ que, durante el periodo de elaboración del plan de estudios y de diseño de las asignaturas del grado se ocupó de establecer el contenido variable de lengua y traducción en el caso de las lenguas $\mathrm{C}$ ( $2^{\mathrm{a}}$ lengua extranjera) y los niveles de lengua en cada una de las asignaturas de lengua extranjera $\left(1^{\circ}\right.$ y $\left.2^{a}\right)$ del plan de estudios, a partir de la adaptación de los niveles de MCER a la formación de traductores FTI-UAB, teniendo en cuenta:

1. Las características filológicas de cada lengua extranjera y su distancia con las lenguas maternas ya que una mayor o menor distancia filológica entre ambas, implica una adquisición de su conocimiento más rápida o más lenta y, por ello, es necesario establecer una progresión en el aprendizaje diferente según cual sea la lengua extranjera.

2. El enfoque textual ya que se trata de formación de lenguas para traductores, de modo que, tanto los objetivos como los resultados de aprendizaje previsibles, están relacionados con

\footnotetext{
${ }^{3}$ La composición de la Comisión para la Implantación, seguimiento y evaluación del grado GTI ha ido variando a lo largo de estos años. Durante el proceso inicial de diseño e implantación del plan de estudios, la comisión estaba formada por las profesoras Marta Arumí, Allison Beeby, Mònica Fernández y Patricia Rodríguez-Inés, y coordinada por Amparo Hurtado Albir. La adaptación y establecimiento de los niveles para las asignaturas del GTI fue el resultado de numerosas reuniones de la comisión con el profesorado de lenguas extranjeras del GTI: Emmanuel Doerr, Helena Aguilà, Maribel Andreu, Mercè Altimir, Regina Saraiva, Paulo Pitta, Albert Branchadell, Yolanda Rodríguez, Allison Beeby, Sara Rovira, Lucía Molina, Albert Nolla, Robert Benda, Agata Orzeszek, Ana de Blas y Lupe Romero.
} 
la traducción de textos y, por tanto, con el desarrollo de competencias textuales.

El resultado de este arduo trabajo fue la concreción y descripción de cada uno de los niveles de entrada y salida de las cuatro competencias fundamentales en la enseñanza de lengua (comprensión escrita-lectora; comprensión oral; producción escrita; producción oral), y para cada una de las asignaturas de lengua extranjera del grado. En el apartado siguiente veremos la adaptación de los niveles MCER a la formación de traductores en el caso concreto de la comprensión lectora.

\section{La adaptación de los niveles de idioma MCER a la formación de lengua para traductores}

El MCER establece tres niveles generales de dominio de la lengua: nivel A (usuario básico); nivel B (usuario independiente) y nivel C (usuario competente) y en cada uno de ellos se describe lo que el usuario sabe hacer en las cuatro habilidades (descriptores para la comprensión lectora, la comprensión oral, la producción escrita y la producción oral). Asimismo, según el sistema escolar o el contexto de aprendizaje, se contempla la posibilidad de establecer subniveles o particiones en la escala mediante numeración (de A1 hasta C2, pasando por A.1.1, A1.2.2., etc.), con sus correspondientes descriptores, sin perder la referencia del objetivo principal del que procede.

Como ya hemos señalado en la introducción, las pruebas realizadas hasta ahora en la FTI se han centrado en la comprensión lectora, por ello y por cuestiones de espacio, en este artículo reportaremos únicamente la adaptación de los niveles de esta competencia y sus descriptores a la formación en lengua extranjera para traductores. En las siguientes tres tablas se ilustra la adaptación efectuada de los niveles A, B y C de comprensión lectora: 


\begin{tabular}{|c|c|c|c|}
\hline \multicolumn{2}{|r|}{$\begin{array}{l}\text { Niveles A - Comprensión lectora } \\
\text { MCER. Escala global }\end{array}$} & \multicolumn{2}{|r|}{ Niveles y descriptores FTI } \\
\hline \multirow[t]{2}{*}{ A1 } & \multirow[t]{2}{*}{$\begin{array}{l}\text { Comprendo palabras y nombres } \\
\text { conocidos y frases muy sencillas, } \\
\text { por ejemplo las que hay en letreros, } \\
\text { carteles y catálogos. }\end{array}$} & Al.1 & $\begin{array}{l}\text { Reconocer el sistema gráfico } \\
\text { y léxico básico y comprender } \\
\text { expresiones escritas básicas } \\
\text { relativas al entomo concreto } \\
\text { e inmediato. }\end{array}$ \\
\hline & & Al.2 & $\begin{array}{l}\text { Comprender información de textos } \\
\text { escritos cortos y sencillos sobre } \\
\text { temas relativos al entorno concreto } \\
\text { e inmediato. }\end{array}$ \\
\hline \multirow[t]{2}{*}{ A2 } & \multirow[b]{2}{*}{$\begin{array}{l}\text { Soy capaz de leer textos muy } \\
\text { breves y sencillos. } \\
\text { Sé encontrar información específica } \\
\text { y predecible en escritos sencillos } \\
\text { y cotidianos como anuncios } \\
\text { publicitarios, prospectos, menús } \\
\text { y horarios y comprendo cartas } \\
\text { personales breves y sencillas. }\end{array}$} & A2.1 & $\begin{array}{l}\text { Comprender textos escritos sencillos } \\
\text { sobre temas cotidianos. }\end{array}$ \\
\hline & & A2.2 & $\begin{array}{l}\text { Comprender textos escritos } \\
\text { sobre temas cotidianos. }\end{array}$ \\
\hline
\end{tabular}

Tabla 1: Niveles A MCER de comprensión lectora. Fonte: Escala global.

\begin{tabular}{|c|l|c|l|}
\hline \multicolumn{2}{|c|}{$\begin{array}{l}\text { Niveles B - Comprensión lectora } \\
\text { MCER. Escala global }\end{array}$} & \multicolumn{2}{c|}{ Niveles y descriptores FTI } \\
\hline B1 & $\begin{array}{l}\text { Comprendo textos redactados en } \\
\text { una lengua de uso habitual y } \\
\text { cotidiano o relacionada con el } \\
\text { trabajo. Comprendo la descripción } \\
\text { de acontecimientos, sentimientos y } \\
\text { deseos en cartas personales. }\end{array}$ & B1.1 & $\begin{array}{l}\text { Comprender textos escritos sobre } \\
\text { temas personales y temas generales } \\
\text { de ámbitos conocidos. }\end{array}$ \\
\cline { 3 - 4 } B2 & $\begin{array}{l}\text { S1.2 } \\
\text { Soy capaz de leer artículos e } \\
\text { informes relativos a problemas } \\
\text { contemporáneos en los que los } \\
\text { autores adoptan posturas o puntos } \\
\text { de vista concretos. Comprendo la } \\
\text { prosa literaria contemporánea } \\
\text { cierta complejidad sobre temas } \\
\text { personales y temas generales } \\
\text { de ámbitos conocidos. }\end{array}$ \\
\cline { 3 - 4 } & $\mathbf{B 2 . 2}$ & $\begin{array}{l}\text { Comprender textos escritos } \\
\text { de tipología diversa con una cierta } \\
\text { complejidad sobre temas generales } \\
\text { de ámbitos conocidos. }\end{array}$ \\
\cline { 3 - 4 } & $\mathbf{B 2 . 3}$ & $\begin{array}{l}\text { Comprender textos escritos } \\
\text { de tipología diversa sobre temas } \\
\text { generales de una amplia gama de } \\
\text { ámbitos y registros. }\end{array}$ \\
\hline
\end{tabular}




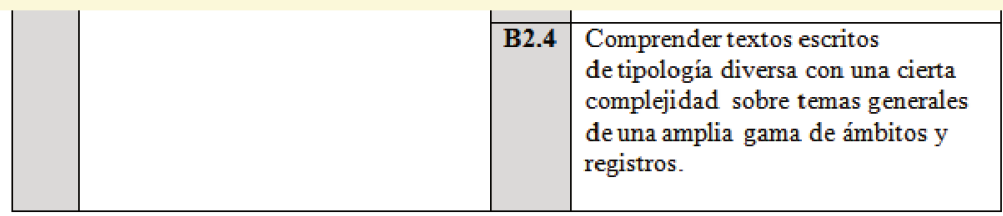

Tabla 2: Niveles B MCER de comprensión lectora. Fonte: Escala global.

\begin{tabular}{|l|l|c|l|}
\hline \multicolumn{2}{|c|}{$\begin{array}{l}\text { Niveles C - Comprensión lectora } \\
\text { MCER. Escala global }\end{array}$} & \multicolumn{2}{|c|}{ Niveles y descriptores FTI } \\
\hline C1 & $\begin{array}{l}\text { Comprendo textos largos } \\
\text { y complejos de carácter literario } \\
\text { obasados en hechos, apreciando } \\
\text { distinciones de estilo. Comprendo } \\
\text { artículos especializados } \\
\text { e instrucciones técnicas largas, } \\
\text { aunque no se relacionen } \\
\text { con mi especialidad. }\end{array}$ & Cl.1 & $\begin{array}{l}\text { Comprender textos escritos } \\
\text { de tipología diversa con una cierta } \\
\text { complejidad de una amplia gama de } \\
\text { ámbitos apreciando las diferencias } \\
\text { estilisticas y geográficas. }\end{array}$ \\
\cline { 3 - 4 } & $\mathbf{C l . 2}$ & $\begin{array}{l}\text { Comprender textos escritos } \\
\text { complejos de tipología diversa de } \\
\text { una amplia gama de ámbitos. } \\
\text { apreciando las diferencias estilisticas } \\
\text { ygeográficas. }\end{array}$ \\
\cline { 3 - 4 } & $\mathbf{C l . 3}$ & $\begin{array}{l}\text { Comprender textos escritos } \\
\text { especializados de tipología diversa } \\
\text { con una cierta complejidad de una } \\
\text { amplia gama de ámbitos. }\end{array}$ \\
\cline { 3 - 4 } & $\mathbf{C l . 4}$ & $\begin{array}{l}\text { Comprender textos escritos } \\
\text { especializados complejos de } \\
\text { tipología diversa de una amplia } \\
\text { gama de ámbitos. }\end{array}$ \\
\hline
\end{tabular}

Tabla 3: Niveles C MCER de comprensión lectora. Fonte: Escala global.

Como puede observarse, de la escala global de niveles MCER, en el GTI se ha procedido a establecer una progresión en cada nivel, mediante la subdivisión en varios niveles, que corresponden a los niveles de comprensión lectora de entrada y salida en las asignaturas del grado. Dichos niveles se reflejan de manera explícita en las guías docentes de las asignaturas, siendo los niveles de entrada los prerrequisitos para cursar la asignatura y los niveles de salida, los objetivos a cumplir en el desarrollo de la misma (véase: http://www.uab.cat/web/estudiar/llistat-de-graus/pla-d- 
estudis/guies-docents/tra duccio-i-interpretacio-1345467811508. html?param1 $=1228291018508$ ) .

\section{Las pruebas de evaluación y verificación de niveles}

En este apartado describiremos el proceso de realización de las pruebas de evaluación y verificación de los niveles de comprensión lectora. Cabe señalar que en este artículo, el término evaluación se utiliza con el significado específico de medición o valoración del nivel de dominio lingüístico que tienen los estudiantes. El objetivo de las pruebas realizadas se circunscribe a evaluar ese aspecto concreto y no otros que, aún siendo muy importantes en la evaluación de un programa de enseñanza de lenguas (como por ejemplo, la eficacia de las metodologías docentes, la idoneidad de los materiales, la satisfacción de estudiante y profesor, etc.), en este estudio no tomamos en consideración.

Desde ese punto de vista, en este apartado daremos cuenta de la realización de: a) la prueba piloto (pruebas del año académico 2011-2012) cuyo resultado sirvió para verificar si los instrumentos y las características de las pruebas funcionaban para la obtención de datos válidos y fiables; b) la segunda prueba (pruebas del año académico 2013-2014) para verificar/contrastar las tendencias y resultados obtenidos en la primera prueba.

a) La prueba piloto: primera verificación de los niveles de lengua extranjera (2011-2012)

En este apartado daremos cuenta de la realización de la prueba piloto, teniendo en cuenta los siguientes factores: características y desarrollo de las pruebas, características de la muestra, instrumento utilizado y resultados obtenidos. 


\section{Características y desarrollo de la pruebas}

La finalidad de las pruebas ha sido la recogida de evidencias de aprendizaje de los siguientes idiomas: inglés, francés, alemán, italiano y portugués de $1^{\circ}, 2^{\circ}$ y $3 r$ curso del GTI. En inglés, francés y alemán se realizaron las pruebas en las asignaturas de primera lengua extranjera (que en la FTI llamamos lengua B) y también de segunda lengua extranjera (que en la FTI llamamos lengua C); en el caso de italiano y portugués, estas dos lenguas extranjeras se imparten sólo como segunda lengua (lengua C).

Las pruebas se realizaron en el segundo semestre del curso académico 2011-2012, desde la coordinación del grado del GTI y bajo la dirección de Emmanuel Doerr se informó previamente a los profesores sobre el manejo del programa y también recibieron instrucciones sobre la recogida de datos. Como ya hemos señalado en la introducción, las pruebas se centraron en verificar si los niveles de competencia lectora alcanzados por los estudiantes, se correspondían a los fijados previamente en el plan de estudios.

Las características de las pruebas fueron las siguientes:

- Se informó a los estudiantes de que se trataba de una prueba de evaluación diagnóstica sin peso en la asignatura.

- La prueba se realizó de forma anónima y de manera voluntaria.

- Solo se evaluó la competencia lectora.

- Se evaluó un único grupo por asignatura (el más numeroso).

- Participaron un máximo de 30 alumnos por grupo (ya que es la capacidad máxima de las aulas multimedia).

Casi todas las pruebas se desarrollaron sin incidencias. Encontramos algunos problemas logísticos que dificultaron la realización de las mismas como la disponibilidad de las aulas multimedias en horario lectivo, que el periodo de las pruebas coincidiera con la época de exámenes, o con el período de encuestas sobre el profesorado. Afortunadamente, estas dificultades fueron solventadas gracias a la colaboración de los estudiantes, del profesorado, y del personal administrativo de la Facultad. 


\section{Características de la muestra}

En esta primera prueba piloto, participaron 274 alumnos de Grado de $1^{\circ}, 2^{\circ}$ y $3 r$ curso y se recogieron datos de 17 de las 21 asignaturas de Idioma B / Idioma C e Idioma y Traducción C (lenguas cercanas) de segundo semestre (las pruebas de cuatro asignaturas coincidieron con huelgas de los estudiantes y no pudieron realizarse por falta de asistencia o datos de menos de 3 estudiantes).

En relación con los estudiantes, cabe destacar que sólo participaron aquellos estudiantes de grado que hubieran aprobado la asignatura precedente el semestre anterior. Esta medida fue tomada para obtener una muestra de estudiantes con una alta probabilidad de haber consolidado las competencias lingüísticas relevantes.

\section{La herramienta: el test dialang}

Para la realización de las pruebas se utilizó el test DIALANG, un sistema de evaluación de la lengua basado en los niveles del MCER, que ha sido desarrollado por más de 20 importantes instituciones con el soporte de la Comisión Europea, para fines de diagnóstico, es decir, para que los estudiantes de lenguas extranjeras sepan cual es el dominio de la lengua que estudian.

El sistema se compone de autoevaluación, pruebas lingüísticas y retroalimentación, que pueden hacerse a través de Internet, y están disponibles en catorce lenguas europeas: alemán, danés, español, finés, francés, griego, holandés, inglés, irlandés, islandés, italiano, noruego, portugués y sueco. No existe ninguna versión para los idiomas ruso, árabe, chino y japonés, de ahí que la verificación de los niveles en estas lenguas no se lleve a cabo con esta herramienta.

Las fases del sistema Dialang de autoevaluación constan de un test inicial léxico y morfológico, cuyos resultados calibran el nivel aproximado en el que se sitúa el estudiante en su dominio del idioma. Acto seguido, se selecciona la competencia que se quiere autoevaluar, que en nuestro caso fue la comprensión lectora. El test de comprensión lectora está formado por una veintena de textos con dificultad progresiva y comprende 30 items o problemas de comprensión textual para medir la comprensión global (10 ítems); 
la identificación de ideas principales (5 ítems); la comprensión detallada (10 items).; y la capacidad de inferencia (5 items). Finalmente, el sistema efectúa una estimación del dominio del estudiante en la competencia seleccionada. Y, desde ese punto de vista, una de las ventajas de este instrumento es que, a diferencia de otros, el resultado de la evaluación no se limita a verificar que se ha alcanzado un nivel determinado (apto/no apto) sino que sitúa al estudiante en un nivel determinado de dominio lingüístico.

\section{Resultados de las prueba piloto}

En las tablas siguientes, ejemplificamos los niveles de comprensión lectora de salida establecidos previamente en el diseño de las asignaturas del GTI, por idioma, curso, y primera o segunda lengua extranjera (idioma B o C en el GTI) y los resultados obtenidos en la prueba piloto:

\begin{tabular}{|l|c|c|c|c|}
\hline \multirow{2}{*}{$\begin{array}{c}\text { Idioma B. } \\
\mathbf{1} \\
\mathbf{1} \\
\text { extranjera }\end{array}$} & \multicolumn{2}{|c|}{ lr curso: asignatura } & \multicolumn{2}{c|}{$\mathbf{2}^{\circ}$ curso: asignatura } \\
\cline { 2 - 5 } & \multicolumn{2}{|c|}{ Idioma B2 } & \multicolumn{2}{c|}{ Idioma B4 } \\
\cline { 2 - 5 } & Guía docente & Test 1 - 2012 & Guía docente & Test 1 - 2012 \\
\hline Alemán & B2.3 & B2.3 & C1.2 & B2.2 \\
\hline Inglés & B2.4 & C1.2 & C1.3 & C1.2 \\
\hline Francés & B2.3 & C1.1 & C1.2 & C1.2 \\
\hline
\end{tabular}

Tabla 4: Resultados de la prueba piloto (test 1): niveles de comprensión lectora en lenguas B ( $1^{\text {a }}$ lengua extranjera). Fonte: Própria

\begin{tabular}{|c|c|c|c|c|c|c|}
\hline \multirow{3}{*}{$\begin{array}{c}\text { Idioma C. } \\
2^{x} \text { lengua } \\
\text { extranjera }\end{array}$} & \multirow{2}{*}{\multicolumn{2}{|c|}{$\begin{array}{c}\text { 1r curso. Asignatura } \\
\text { Idioma C2 }\end{array}$}} & \multirow{2}{*}{\multicolumn{2}{|c|}{$\frac{2^{\circ} \text { curso. Asignatura }}{\text { IDTR C2 }}$}} & \multirow{2}{*}{\multicolumn{2}{|c|}{$\frac{3^{\circ} \text { curso. Asignatura }}{\text { IDTRC4 }}$}} \\
\hline & & & & & & \\
\hline & $\begin{array}{c}\text { Guía } \\
\text { docente }\end{array}$ & $\begin{array}{l}\text { Test l } \\
2012\end{array}$ & $\begin{array}{c}\text { Guía } \\
\text { docente }\end{array}$ & $\begin{array}{l}\text { Test 1 } \\
2012\end{array}$ & $\begin{array}{c}\text { Guía } \\
\text { docente }\end{array}$ & $\begin{array}{l}\text { Test 1 } \\
2012\end{array}$ \\
\hline Alemán & A2.2 & A2.2 & B2.1 & B1.1 & B2.3 & $\mathrm{A} 2.2$ \\
\hline Inglés & B1.2 & B2.2 & B2.3 & $\varnothing$ & C1.2 & $\varnothing$ \\
\hline Francés & B1.2 & $\mathrm{Cl} .2$ & B2.3 & $\mathrm{Cl.2}$ & C1.2 & Cl.2 \\
\hline Italiano & B1.2 & B2.1 & B2.3 & B2.3 & C1.2 & $\varnothing$ \\
\hline Portugués & B1.2 & Cl.1 & B2.3 & Cl.1 & C1.2 & $\varnothing$ \\
\hline
\end{tabular}

Tabla 5: Resultados de la prueba piloto (test 1): niveles de comprensión lectora en lenguas $\mathrm{C}\left(2^{\mathrm{a}}\right.$ lengua extranjera). Fonte: Própria 
De los datos de esta primera prueba puede observarse que la gran mayoría de asignaturas $(76,4 \%)$ presentaba un nivel superior o equivalente respecto a los niveles de salida fijados previamente en las guías del GTI (datos con sombreado verde y azul respectivamente). De hecho únicamente 4 asignaturas de las 17 evaluadas presentaban niveles inferiores (datos en naranja).

Respecto a los resultados por lenguas, cabe destacar que, a excepción de la asignatura de inglés de $2^{\mathrm{a}}$, (que no alcanzaba el nivel fijado en la guía pero con una diferencia mínima " $\mathrm{C} 1.2$ " frente a C1.3") los datos con niveles claramente inferiores correspondían a asignaturas de alemán (como primera y como segunda lengua extranjera). Este dato ya nos indicaba que existe una progresión diferente en la adquisión de esta lengua respecto al resto de idiomas sujetos a anàlisis.

En relación con los resultados por primera o segunda lengua extranjera (lenguas B o C) podemos destacar que, a excepción del alemán, en todas las lenguas se producían niveles equivalentes o superiores de comprensión lectora en el primer año mientras que en $2^{\circ}$ y $3^{\circ}$, aún manteniéndose en los niveles fijados por el GTI, se reflejaba un cierto estancamiento en la progresión del dominio de la comprensión lectora debido, probablemente, a la reducción en horas de lengua que ha supuesto la implantación del grado respecto a la anterior licenciatura.

A partir de los datos obtenidos en la prueba piloto verificamos los siguientes aspectos:

1. El test Dialang resultó ser un instrumento eficaz para evaluar los niveles de comprensión lectora porque nos permitía medir de manera fiable la comprensión lectora en las lenguas interesadas.

2. En líneas generales, los niveles de comprensión lectora se ajustaban o superaban los niveles fijados por el GTI. Obviamente, se necesitaban más pruebas para verificar si se trataba de una tendencia que se mantenía en el tiempo, o eran resultados coyunturales que tenían que ver con circuns- 
tancias puntuales del año académico en cuestión.

3. Existían diferencias en la progresión del aprendizaje incluso entre lenguas que a priori pueden ser consideradas "cercanas", ya que, aunque el alemán no es una lengua filológicamente distante del español como pueda serlo el árabe o el ruso, los datos obtenidos parecían indicar que su adquisión es más lenta que en otras lenguas cercanas o que requiere de un mayor número de horas de lengua para llegar a los niveles fijados por el GTI.

\section{b) La segunda prueba: verificación de los niveles de lengua extranjera (2012-2014)}

En esta segunda prueba partíamos de las conclusiones anteriores $\mathrm{y}$, por tanto, aparte de seguir contando con el test DIALANG para medir el nivel de comprensión lectora de los estudiantes, y continuar con los mismos criterios de selección de la muestra, nuestro objetivo principal era verificar las tendencias observadas en relación con la igualación y/o superación de los niveles en la mayoría de asignaturas y la tendencia a no alcanzar los niveles fijados en las asignaturas de alemán. Además, a diferencia de la prueba anterior, también podíamos realizar las pruebas en el cuarto año, y este factor nos aportaba información acerca de los niveles de comprensión lectora de los estudiantes al finalizar sus estudios.

En esta ocasión, participaron 303 alumnos de Grado de $1^{\circ}, 2^{\circ}$, $3^{\mathrm{a}}$ y $4^{\mathrm{a}}$ curso y se recogieron datos de 24 de las 26 asignaturas de Idioma B / Idioma C e Idioma y Traducción C (lenguas cercanas) de segundo semestre. En las siguientes tablas se recogen los resultados de las pruebas de la segunda prueba en comparativa con los resultados obtenidos en la prueba piloto y los niveles fijados en las guías docentes: 


\begin{tabular}{|c|c|c|c|c|c|c|}
\hline \multirow{3}{*}{$\begin{array}{c}\text { Idioma B. } \\
1^{\mathrm{a}} \text { lengua } \\
\text { extranjera }\end{array}$} & \multicolumn{3}{|c|}{ lr curso. Asignatura } & \multicolumn{3}{|c|}{$2^{\circ}$ curso. Asignatura } \\
\hline & \multicolumn{3}{|c|}{ Idioma B2 } & \multicolumn{3}{|c|}{ Idioma B4 } \\
\hline & $\begin{array}{c}\text { Guía } \\
\text { docente }\end{array}$ & $\begin{array}{l}\text { Test 1 } \\
2012\end{array}$ & $\begin{array}{l}\text { Test 2 } \\
2014\end{array}$ & $\begin{array}{c}\text { Guía } \\
\text { docente }\end{array}$ & $\begin{array}{l}\text { Test 1 } \\
2012\end{array}$ & $\begin{array}{l}\text { Test } 2 \\
2014\end{array}$ \\
\hline Alemán & B2.3 & B2.3 & B2.1 & C1.2 & B2.2 & $\mathrm{Cl} .1$ \\
\hline Inglés & B2.4 & $\mathrm{C} 1.2$ & $\mathrm{Cl} 1.2$ & C1.3 & $\mathrm{Cl} 1.2$ & B2.4 \\
\hline Francés & B2.3 & $\mathrm{Cl} .1$ & Cl.3 & C1.2 & $\mathrm{C} 1.2$ & $\mathrm{Cl} 1.3$ \\
\hline
\end{tabular}

Tabla 6: Resultados de las dos pruebas (Test 1 y Test 2): niveles de comprensión lectora en lenguas B ( $1^{\text {a }}$ lengua extranjera). Fonte: Própria

\begin{tabular}{|c|c|c|c|c|c|c|c|c|c|c|c|c|}
\hline \multirow{2}{*}{$\begin{array}{l}\text { Idioma C. } \\
2^{2} \text { Lengua } \\
\text { Extranjera }\end{array}$} & \multicolumn{3}{|c|}{ 1r curso. Asignatura } & \multicolumn{3}{|c|}{$\begin{array}{c}2^{\circ} \text { curso. Asignatura } \\
\text { Idioma y traducción C2 }\end{array}$} & \multicolumn{3}{|c|}{$\begin{array}{l}\text { 3r curso. Asignatura } \\
\text { Idiom a traducción C4 }\end{array}$} & \multicolumn{3}{|c|}{$4^{\circ}$ curso. Asignatura } \\
\hline & Guí & $\begin{array}{l}\text { dioma C } \\
\text { Test 1 } \\
2012 \\
\end{array}$ & $\begin{array}{l}\text { Test } 2 \\
2014 \\
\end{array}$ & $\begin{array}{l}\text { Idion } \\
\text { Guia }\end{array}$ & $\begin{array}{l}\text { traduc } \\
\text { Test 1 } \\
2012 \\
\end{array}$ & $\begin{array}{l}\text { on C2 } \\
\text { Test } 2 \\
2014\end{array}$ & $\begin{array}{l}\text { Idio } \\
\text { Guia }\end{array}$ & $\begin{array}{l}y \text { trad } \\
\text { Test } 1 \\
2012\end{array}$ & $\begin{array}{l}\text { ión C4 } \\
\text { Test } 2 \\
2014 \\
\end{array}$ & $\begin{array}{l}\text { Idion } \\
\text { Guía }\end{array}$ & $\begin{array}{l}\text { traduc } \\
\text { Test } 1 \\
2012\end{array}$ & $\begin{array}{l}\text { ón C6 } \\
\text { Test } 2 \\
2014 \\
\end{array}$ \\
\hline Dlemán & 2.2 & $A 2.2$ & A2.2 & B2.1 & B1.1 & B1.1 & B2.3 & $A 2.2$ & $\mathrm{~B} 1.2$ & $\mathrm{C} 1.2$ & $\varnothing$ & $\mathrm{B} 1.2$ \\
\hline Inglés & 31.2 & B2.2 & $\varnothing$ & B2.3 & $\varnothing$ & $\varnothing$ & C1.2 & $\varnothing$ & $\mathrm{Cl} .4$ & C1.4 & $\varnothing$ & B2.3 \\
\hline Francés & 1.2 & $\mathrm{Cl} .2$ & $\mathrm{Cl} .2$ & B2.3 & $\mathrm{Cl} .2$ & $\mathrm{Cl} .2$ & C1.2 & $\mathrm{Cl} .2$ & $\mathrm{Cl} .4$ & C1.4 & $\varnothing$ & $\mathrm{Cl} .2$ \\
\hline Italiano & B1.2 & B2.1 & B2.2 & B2.3 & B2.3 & B2.3 & C1.2 & $\varnothing$ & B2.3 & C1.4 & $\varnothing$ & $\mathrm{Cl} .2$ \\
\hline Portugués & B1.2 & $\mathrm{Cl} . \mathrm{I}$ & B2.3 & B2.3 & $\mathrm{Cl} . \mathrm{I}$ & $\mathrm{Cl} . \mathrm{I}$ & C1.2 & $\mathrm{Cl} .3$ & B2.1 & C1.4 & $\varnothing$ & $\mathrm{Cl} .2$ \\
\hline
\end{tabular}

Tabla 7: Resultados de las dos pruebas (Test 1 y Test 2): niveles de comprensión lectora en lenguas $\mathrm{C}\left(2^{\mathrm{a}}\right.$ lengua extranjera). Fonte: Própria

En relación con los resultados de la lengua $\mathrm{B}$, podemos constatar que se confirma la tendencia de superar los niveles en el caso de las asignaturas de francés, de estancamiento en segundo curso de inglés y de no alcanzar los niveles de alemán en ninguno de los dos cursos.

En el caso de las lenguas $\mathrm{C}$, si nos atenemos a los resultados por curso, podemos observar que:

- En primero se mantiene la tendencia que habíamos observado en la prueba piloto de superar los niveles fijados en las guías en francés, italiano y portugués, y en igualar el nivel fijado en la guía para alemán.

- En segundo se mantiene la misma tendencia que habíamos observado en las primeras pruebas, de superar los niveles fijados en las guías en francés y portugués, de igualar los niveles fijados en italiano y de no alcanzar, en el caso del alemán, el nivel fijado en la guía. 
- En tercero, curso en el que sólo se imparte traducción, se mantiene la tendencia de estancamiento acentuándose más y en más lenguas. En el caso del alemán se mantiene en un nivel inferior del fijado en la guía, y el italiano y el portugués tampoco alcanzan los niveles mínimos; las únicas lenguas que igualan y superan el nivel son el inglés y el francés.

- En cuarto, ninguna de las lenguas $\mathrm{C}$ alcanza los niveles fijados en las guías. Si bien en el caso de francés, italiano y portugués los niveles alcanzados permiten una comprensión lectora de textos complejos, en el caso de inglés y sobre todo del alemán no hay un cambio significativo en los niveles alcanzados en $2^{\circ}$ y $3 \mathrm{r}$ curso, y por tanto, se mantiene el estancamiento observado en tercero en la progresión en la comprensión lectora.

\section{Conclusiones}

De estos primeros resultados podemos concluir que:

1. Se confirma una clara diferencia en la progresión de la comprensión lectora entre el alemán y el resto de lenguas denominadas "cercanas" (francés, inglés, italiano, portugués) puesto que tanto en lengua $\mathrm{B}$ como en lengua $\mathrm{C}$, las previsiones de niveles de salida de las asignaturas no se cumplen y quedan muy por debajo de los niveles establecidos. Parece ser que el alemán, pese a tener el mismo alfabeto que las lenguas cercanas y las lenguas A (español y catalán), presenta una progresión más similar a lenguas con alfabetos diferentes como el árabe, el ruso, el chino o el japonés.

2. Se confirma un cierto estancamiento en la progresión de la mayoría de lenguas $\mathrm{C}$ en tercer curso que se mantiene en cuarto curso. Recordemos que en tercer curso todos los contenidos de las asignaturas de lengua $\mathrm{C}$ se dedican exclusivamente a la traducción, habrá que considerar si este aspecto 
incide en el estancamiento en la progresión o ésta se debe a otros factores, ya que no sucede en todos los casos.

Obviamente, tratándose de los primeros resultados de dos años académicos, estas conclusiones deben interpretarse como tendencias, algunas de ellas, como en el caso de la lengua alemana, muy significativas. No obstante, aunque los datos obtenidos sean indicadores de la situación, no podemos extraer conclusiones generalizables.

Son datos de pruebas que miden los niveles de dos años concretos y no consecutivos, cuyos valores pueden variar al siguiente, según el nivel inicial medio del grupo, las horas efectivas de clase, el input pedagógico, etc. Para corroborar las tendencias encontradas y evitar extraer conclusiones erróneas por prematuras o por falta de contraste, habrá que comparar los datos obtenidos en secuencia consecutiva con los resultados de pruebas futuras.

\section{Referências}

BREHM, J. Developing Foreign Language Reading Skill in Translator Trainees. Tesis doctoral. Universitat Autònoma de Barcelona, 1997.

BREHM CRIPPS, J.; HURTADO ALBIR, A. La enseñanza de lenguas en la formación de traductores. In: HURTADO ALBIR, A. (Ed.). Enseñar a traducir: metodología en la formación de traductores e intérpretes. Madrid: Edelsa, 1999.

BERENGUER, L. Didáctica de segundas lenguas en los estudios de traducción. In: HURTADO ALBIR, A. (Ed.). La enseñanza de la traducción. Castellón: Universitat Jaume I, 1996. Col. Estudis sobre la Traducció 3.

. L'ensenyament de llengües estrangeres per a traductors. Didàctica de l'alemany. Tesis doctoral. Universitat Autònoma de Barcelona, 1997.

Cad. Trad., Florianópolis, v. 38, n $^{0}$ 2, p. 320-338, mai-ago, 2018 
COUNCIL OF EUROPE. Common European Framework of Reference for Languages: Learning, Teaching, Assessment. Strasbourg, 2001. Versión española on-line: < http://cvc.cervantes.es/ensenanza/biblioteca_ele/marco/default.htm > .

Recebido em: 13 de dezembro de 2017 Aceito em: 29 de fevereiro de 2018 Publicado em: maio de 2018

Lupe Romero. E-mail: luperomero@uab.cat 